\title{
Initial Conditions in String Cosmology
}

\author{
Dominic Clancy ${ }^{1 a}$, James E. Lidsey ${ }^{2 b} \&$ Reza Tavakol $^{1 c}$
}

${ }^{1}$ Astronomy Unit, School of Mathematical Sciences, Queen Mary $\mathcal{E}_{3}$ Westfield College, Mile End Road, LONDON, E1 4NS, U.K.

${ }^{2}$ Astronomy Centre and Centre for Theoretical Physics, University of Sussex, BRIGHTON, BN1 9QJ, U.K.

\begin{abstract}
We take a critical look at a recent conjecture concerning the past attractor in the pre-big-bang scenario. We argue that the Milne universe is unlikely to be a general past attractor for such models and support this with a number of examples.
\end{abstract}

PACS NUMBERS: $98.80 . \mathrm{Cq}$

${ }^{a}$ Electronic address: dominic@maths.qmw.ac.uk

${ }^{b}$ Electronic address: jel@astr.cpes.susx.ac.uk

${ }^{c}$ Electronic address: reza@maths.qmw.ac.uk 
An important issue in cosmology, whether in the context of general relativity or string theory, is that of "naturalness". This in turn translates into the question of initial conditions. One way of studying this question is to ask whether there exists an "attractor" whose basin of attraction (i.e. the set of initial conditions which evolve to this state) has a large or full measure in the space of all possible initial data.

In its fullness, this is an impossible question to address at present for a number of reasons. Despite dramatic recent progress in our understanding of M-theory, we still lack a definitive non-perturbative formulation of string theory. (For a recent review, see, e.g., Ref. [1]). This raises the question of whether the results obtained within the context of the perturbative effective actions are representative and robust with respect to higher-derivative and loop corrections. Furthermore, the nature of the generic attractor is not known even at this reduced level because the resulting equations are non-linear partial differential equations. Consequently, additional restrictions such as spatial homogeneity must be imposed to drastically reduce the complexity of these equations. The crucial point here is to establish those attractors that have the largest basins and are therefore the most 'natural' at this level of approximation.

An interesting recent development within string cosmology has been the pre-bigbang scenario [2]. In this picture, an accelerated, inflationary expansion is driven by the rapid increase of the gravitational (string) coupling, as parametrized by the dilaton field. The fundamental postulate of the pre-big-bang cosmology is that the initial data for inflation lies well within the perturbative regime, where the curvature and coupling are very small. Inflation then proceeds for sufficiently homogeneous initial conditions, where time derivatives are dominant with respect to spatial gradients, and the universe evolves into a high curvature and strongly-coupled regime. At the level of the lowest-order effective action, the final state of this evolution is singular in both the curvature and coupling, but it has been proposed that higher-order corrections should become important at the string scale [3].

The behaviour of pre-big-bang cosmology in the asymptotic past before the onset

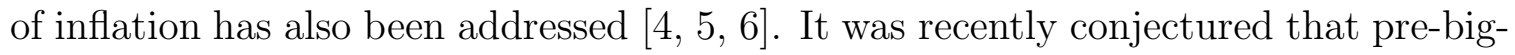
bang inflation generically evolves out of an initial state that approaches the Milne universe in the semi-infinite past, $t \rightarrow-\infty$, where $t$ represents synchronous time [5]. The Milne universe may be mapped onto the future (or past) light cone of the origin of Minkowski spacetime and therefore corresponds to a non-standard representation of the string perturbative vacuum. It is flat spacetime expressed in an expanding frame:

$$
d s^{2}=-d t^{2}+t^{2}\left(d x^{2}+e^{-2 x}\left(d y^{2}+d z^{2}\right)\right) .
$$

The proposal is that the Milne background represents an early time attractor, with a large measure in the space of initial data [5]. If so, this would provide strong justification for the postulate that inflation begins in the weak coupling and curvature regimes and would render the pre-big-bang assumptions regarding the initial states as 'natural'.

The aim of this paper is to take a careful look at this conjecture. Since pre-big 
bang inflation must begin in the perturbative regime, the dynamics of the universe is well approximated by the string effective action. To lowest-order in the inverse string tension, the massless bosonic excitations in the Neveu-Schwarz/Neveu-Schwarz sector of the theory are the dilaton, $\Phi$, the graviton, $g_{\mu \nu}$, and the anti-symmetric two-form potential, $B_{\mu \nu}$, with field strength, $H_{\mu \nu \lambda} \equiv \partial_{[\mu} B_{\nu \lambda]}$. The effective action is [7]

$$
S=\int d^{4} x \sqrt{-g} e^{-\Phi}\left[R+(\nabla \Phi)^{2}-\frac{1}{12} H_{\mu \nu \lambda} H^{\mu \nu \lambda}\right],
$$

where $R$ is the Ricci curvature scalar and $g \equiv \operatorname{det} g_{\mu \nu}$.

Fundamental strings sweep geodesic surfaces with respect to the string frame metric, $g_{\mu \nu}$, but it is convenient to discuss the dynamics in the conformally related Einstein frame, where the dilaton is minimally coupled to the graviton:

$$
\tilde{g}_{\mu \nu} \equiv e^{-\Phi} g_{\mu \nu}
$$

Moreover, the field strength of the two-form potential is dual to a one-form in four dimensions, i.e., $H^{\alpha \beta \gamma} \equiv e^{\Phi} \epsilon^{\alpha \beta \gamma \delta} \nabla_{\delta} \sigma$, where $\epsilon^{\alpha \beta \gamma \delta}$ is the covariantly constant fourform. This one-form may then be interpreted as the field strength of a pseudo-scalar axion field, $\sigma$. The effective action (2) is therefore equivalent to

$$
S=\int d^{4} x \sqrt{-\tilde{g}}\left[\tilde{R}-\frac{1}{2}(\tilde{\nabla} \Phi)^{2}-\frac{1}{2} e^{2 \Phi}(\tilde{\nabla} \sigma)^{2}\right]
$$

For the class of models we consider, a massless, minimally coupled scalar field $\phi$ (where the gradient of the scalar field is a timelike vector) may be interpreted in terms of a stiff perfect fluid:

$$
\begin{aligned}
p=\rho & =\frac{1}{2} \phi_{, \alpha} \phi^{, \alpha} \\
u_{\alpha} & =\frac{\phi_{, \alpha}}{\left(\phi_{, \beta} \phi^{, \beta}\right)^{1 / 2}},
\end{aligned}
$$

where $p$ is the pressure, $\rho$ is the energy density and $u_{\alpha}$ is the fluid four-velocity. Since both the dilaton and axion fields are massless, the energy-momentum tensor in the Einstein frame is then equivalent to that of a stiff perfect fluid, with the equation of state $p=\rho$.

In the synchronous frame, where $g_{00}=-1$ and $g_{0 i}=0$, the spacetime metric may be written in the form $d s^{2}=-d t^{2}+h_{i j} d x^{i} d x^{j}(i, j=1,2,3)$. The Einstein field equations in this frame are then given by [8]

$$
\begin{aligned}
R_{0}^{0} & =-\frac{1}{2} \frac{\partial}{\partial t} \chi_{i}^{i}-\frac{1}{4} \chi_{i}^{j} \chi_{j}^{i}=T_{0}^{0}-\frac{1}{2} T \\
R_{i}^{0} & =\frac{1}{2}\left(\chi^{j}{ }_{i ; j}-\chi^{j}{ }_{j ; i}\right)=T_{i}^{0} \\
R_{i}^{j} & =-{ }^{(3)} R_{i}^{j}-\frac{1}{2 \sqrt{h}} \frac{\partial}{\partial t}\left(\sqrt{h} \chi_{i}^{j}\right)=T_{i}^{j}-\frac{1}{2} \delta_{i}^{j} T
\end{aligned}
$$


where $\chi_{i j} \equiv \partial h_{i j} / \partial t,{ }^{(3)} R_{j}^{i}$ is the Ricci curvature tensor constructed from the threemetric $h_{i j}, h \equiv \operatorname{det} h_{i j}$, a semi-colon denotes covariant differentiation with respect to $h_{i j}$ and units are chosen such that $8 \pi G=1$.

We begin by briefly reviewing the argument that the only non-singular fixed point of the Einstein field equations (77)-(9) with a massless scalar field is flat space [1, 5]. It can be shown that the fixed points exist either at infinity or at $\dot{\phi}=\chi_{i j}=\nabla^{i} \nabla_{i} \phi=0$. These two possibilities correspond to a singularity or a stationary field, respectively. The latter further implies that $\nabla_{i} \phi=0$ if boundary terms are neglected and it then follows from the field equations that ${ }^{(3)} R_{i j}=0$. Since in three dimensions a vanishing Ricci tensor implies a vanishing Riemann tensor, it is concluded that the only non-singular past attractor corresponds to flat spacetime. Buonanno et al. [5] then conjecture that those pre-big-bang models that are negatively-curved and sufficiently isotropic generically evolve out of the Milne state.

However, we recall that an important feature of synchronous reference frames is that they are manifestly not stationary, as emphasized by Landau and Lifshitz [8]. In other words, a gravitational field can not be constant in such a frame (i.e. of the form $\chi_{i j}=0$ ) while possessing a non-zero energy-momentum tensor, as can be seen directly from Eq. (7). Thus, synchronous frameworks are not compatible with non-vacuum, stationary fields (fixed points). As a result, the Milne universe as a seemingly sole past attractor arises as a direct consequence of working in the synchronous frame, because in this frame the only non-singular stationary solution (fixed point) is necessarily flat space.

Now, given that generically one expects to have a non-zero energy-momentum tensor at the onset of inflation, a more natural question is to ask whether the Milne universe is likely to be the past attractor with the largest measure of initial states if one starts with a non-vacuum, pre-big-bang universe and runs it backwards towards $t \rightarrow-\infty$. In the following we shall argue that this is unlikely. We should emphasize here that the Milne universe may still be an attractor for a certain class of cosmologies. The question we address is that of its likelihood (i.e. the size of the corresponding basin and whether or not it carries a full measure).

To substantiate the above discussion, we proceed to present some concrete examples for which the past attractor is not the Milne universe. We consider families of models that are often considered in theoretical cosmology, and ask what is the generic initial state among these particular sets of models out of which an inflating pre-big-bang cosmology evolved.

Without loss of generality, we may work in the Einstein frame, since we are interested in asymptotic states where the dilaton and axion fields approach constant values. In this case, the string and Einstein frames become equivalent. It then follows from the time symmetry of the Einstein field equations that we may gain insight into the nature of the past attractor of pre-big-bang cosmology by instead considering the future asymptotic states of classical cosmological solutions to Einstein gravity containing a stiff perfect fluid. 
We begin with the spatially homogeneous Bianchi models. (For a review of the Bianchi classification scheme, see, e.g., Ref. [9]). These universes admit a threedimensional Lie group of isometries acting simply transitively on the spacelike hypersurfaces, $t=$ constant. In the Ellis-MacCallum classification, the models are separated into two classes, $\mathrm{A}$ and $\mathrm{B}$, depending on the specific group type [10]. Bianchi models are referred to as orthogonal cosmologies when the fluid flow is orthogonal to the group orbits. Otherwise they represent 'tilted' models. Tilted models are homogeneous to observers with world lines directed orthogonally to the $t=$ constant hypersurfaces, but appear to be inhomogeneous to those observers that comove with the fluid.

It is natural to suppose that the ability of a given model to approach the vacuum Milne state at late times should be related to the question of whether it is able to isotropize. Collins and Hawking [11] have proved a theorem stating that modulo some very general assumptions about the matter fields (that are satisfied by an orthogonal stiff fluid), the set of spatially homogeneous models that isotropize at late times is of measure zero in the space of homogeneous initial data. Indeed, they prove that the only Bianchi types that could possibly isotropize at arbitrarily late times are the types $\mathrm{I}, \mathrm{V}, \mathrm{VII}_{0}$ and $\mathrm{VII}_{h}$. It turns out that types $\mathrm{I}, \mathrm{V}$ and $\mathrm{VII}_{0}$ can approach isotropy. However, the type $\mathrm{VII}_{h}$, which is the only model amongst this subgroup that has non-zero measure in the space of all homogeneous models, in general does not. In fact, a necessary condition for a type $\mathrm{VII}_{h}$ model to isotropize is that it must tend to a $k=-1$ Friedmann-Robertson-Walker (FRW) universe. Even though this model does approach Milne asymptotically, it is nevertheless of measure zero in the set of $\mathrm{VII}_{h}$ models. This suggests that the set of type $\mathrm{VII}_{h}$ pre-big-bang models driven by a dilaton field that would have evolved out of the Milne state is of measure zero in the space of type $\mathrm{VII}_{h}$ cosmologies.

We now consider the class B models in more detail. This class contains the Bianchi types IV, $\mathrm{V}, \mathrm{VI}_{h}$ and $\mathrm{VII}_{h}$. It is known that all orthogonal class B perfect fluid models expand indefinitely into the future $(t>0)$. Hewitt and Wainwright have proved an important theorem for these models by employing a dynamical systems approach to homogeneous cosmology [12]. They have shown that all orthogonal class B Bianchi models with a stiff perfect fluid, apart from a set of measure zero, are asymptotic in the future to a plane wave state and asymptotic in the past to the Jacobs Bianchi I solution. We may interpret this result in the pre-big-bang context by interchanging the past and future states. The Jacobs stiff perfect fluid solution then describes the evolution of the universe towards the singularity [13]. It contains two essential parameters and may be interpreted as the generalization of the vacuum Kasner solution to include a dilaton field [14]. It is precisely this solution that corresponds to dilaton-driven, pre-big-bang inflation in the string frame when the parameters satisfy appropriate conditions [15]. In general, however, the initial state does not correspond to a region of flat space, but rather to a plane wave. Two exceptions are the orthogonal types $\mathrm{V}$ and $\mathrm{VI}_{-1}$. (The latter model is sometimes referred to as the type III). 
In these cases, the models do indeed approach flat space as we trace their behaviour back to $t \rightarrow-\infty$.

Further insight may be gained by considering the type $\mathrm{VII}_{h}$ model. In an appropriate coordinate frame, the most general type $\mathrm{VII}_{h}$ metric may be written in the form 16

$$
d s^{2}=a^{-2}\left(-d \tau^{2}+d x^{2}\right)+e^{2(\lambda-x)} d S^{2}
$$

where

$$
\begin{aligned}
d S^{2}= & \cosh \mu\left(d y^{2}+d z^{2}\right) \\
& -\sinh \mu\left[\left(d z^{2}-d y^{2}\right) \cos 2(k x+\varphi)+2 d y d z \sin 2(k x+\varphi)\right],
\end{aligned}
$$

the four variables $\{a, \mu, \lambda, \varphi\}$ are functions only of $t, k^{2} \equiv h^{-1}$ and we have defined a time parameter $\tau \equiv \int^{t} d t_{1} a\left(t_{1}\right)$. The Einstein field equations (7)-(9) containing an orthogonal perfect fluid are given by [17]

$$
\begin{gathered}
\lambda^{\prime \prime}+2 \lambda^{\prime 2}-2-\frac{1}{2}(\rho-p)=0, \\
\nu^{\prime}+2 \lambda^{\prime} \nu+2 \sinh 2 \mu\left(k^{2}-\varphi^{\prime 2}\right)=0, \\
\left(\varphi^{\prime} \sinh ^{2} \mu\right)^{\prime}+2 \lambda^{\prime}\left(\varphi^{\prime} \sinh ^{2} \mu\right)+2 k \sinh ^{2} \mu=0, \\
\alpha^{\prime}+2 \alpha \lambda^{\prime}+2\left(1+k^{2} \sinh ^{2} \mu\right)+\frac{1}{2}(\rho-p)=0, \\
\alpha+\lambda^{\prime}-k \varphi^{\prime} \sinh ^{2} \mu=0, \\
\frac{1}{4} \nu^{2}+\left(3+k^{2} \sinh ^{2} \mu\right)\left(1-\lambda^{\prime 2}\right)+\sinh ^{2} \mu\left(k \lambda^{\prime}+\varphi^{\prime}\right)^{2}+\rho=0,
\end{gathered}
$$

where $\nu \equiv \mu^{\prime}, \alpha \equiv a^{\prime} / a$, a prime denotes differentiation with respect to $\tau$ and the energy density and pressure have been scaled by a factor $a^{-2}$.

These equations for a stiff perfect fluid are identical to those of the vacuum model, with the exception of Eq. (17), where there is an additional term due to the energy density. This allows the asymptotic form of the general type $\mathrm{VII}_{h}$ stiff perfect fluid solution to be established [16, 17]. Eq. (12) can be solved in full generality and the solution approaches $\lambda^{\prime} \rightarrow 1$ as $\tau \rightarrow \infty$. Thus, the second term on the left-hand side of Eq. (17) vanishes, but since the remaining terms in this equation are all positive definite, they must each vanish separately. It follows, therefore, that $\rho \rightarrow 0, \varphi^{\prime} \rightarrow-k$, $\mu \rightarrow \mu_{0}=$ constant and $\alpha \rightarrow\left(1+k^{2} \sinh ^{2} \mu\right)$. This metric corresponds to the vacuum type $\mathrm{VII}_{h}$ plane wave solution first derived by Doroshkevich, Lukash and Novikov [18]. It may be interpreted as representing two monochromatic, circularly-polarized gravitational waves traveling with constant amplitudes in opposite directions along the $x$-axis [19]. This plane wave solution would have to isotropize if it were to approach the Milne state at large $t$, but it is known that this does not occur. Thus, all but a set 
of measure zero orthogonal stiff perfect fluid type $\mathrm{VII}_{h}$ cosmologies asymptotically approach a plane wave and can not be written in the Milne form. An example of a type $\mathrm{VII}_{h}$ solution that does tend to Milne is the particular $h=4 / 11$ solution due to Barrow 20].

It is important to note that the orthogonal models are special. It is therefore pertinent to consider the effect that tilt has on the asymptotic behaviour of the class $\mathrm{B}$ models. This is interesting because tilt can be viewed as a form of inhomogeneity. Of particular interest is the type $\mathrm{V}$ model, since the orthogonal stiff fluid solution does approach the Milne model [21]. We consider the tilted type V stiff fluid solution found by Maartens and Nel [22]:

$$
\begin{aligned}
d s^{2} & =e^{2 k}\left(-d \tau^{2}+d x^{2}\right)+r\left(f d y^{2}+f^{-1} d z^{2}\right) \\
\rho & =e^{-2 k}\left({\sigma_{, \tau}}^{2}-\sigma_{, x}{ }^{2}\right)
\end{aligned}
$$

where a comma denotes partial differentiation, the functions $\{r, f, k, \sigma\}$ are defined by

$$
\begin{aligned}
r & =e^{-2 q x} \sinh 2 q \tau \\
f & =(\tanh q \tau)^{m} \\
e^{2 k} & =(\sinh 2 q \tau)^{\alpha^{2}+\beta^{2}+\left(m^{2}-1\right) / 2}(\tanh q \tau)^{2 \alpha \beta} \\
e^{\sigma} & =(\tanh q \tau)^{-\alpha}(\sinh 2 q \tau)^{-\beta} e^{2 \beta q x},
\end{aligned}
$$

and the constants $\{m, \alpha, \beta\}$ satisfy the constraint

$$
m^{2}-3+2\left(\alpha^{2}-\beta^{2}\right)=0 .
$$

The scalar function $\sigma$ may be interpreted as a minimally coupled scalar field. The solution is tilted if $\beta \neq 0$ and the fluid flow is orthogonal to the surfaces of homogeneity if $\beta=0$.

In the asymptotic limit $\tau \rightarrow \infty(t \rightarrow \infty)$, the metric reduces to

$$
d s^{2}=e^{2\left(2 \beta^{2}+1\right) \tau}\left(-d \tau^{2}+d x^{2}\right)+e^{2 \tau-2 x}\left(d y^{2}+d z^{2}\right),
$$

where we have specified $q=1$ without loss of generality. The matter lines become null and this corresponds to the case of extreme tilt. Eq. (22) is a locally rotationally symmetric (LRS) model and can be identified with the equilibrium point labeled $\mathcal{H}$ in the notation of Hewitt and Wainwright [21]. It can be shown that for this model the dimensionless variable defined by $\Sigma_{+} \equiv \sigma_{+} / \theta$, where $\sigma_{+}$is a shear parameter and $\theta$ is the rate of expansion scalar, tends to a non-vanishing constant given by $\Sigma_{+}=-2 \beta^{2} /\left(3+2 \beta^{2}\right)$.

Moreover, if we define the light-cone coordinates:

$$
\begin{aligned}
u & \equiv \exp \left[\left(1+2 \beta^{2}\right)(\tau-x)\right] \\
v & \equiv \frac{1}{\left(1+2 \beta^{2}\right)^{2}} \exp \left[\left(1+2 \beta^{2}\right)(\tau+x)\right] .
\end{aligned}
$$


Eq. (22) may be written in the form

$$
d s^{2}=-d u d v+u^{2 /\left(1+2 \beta^{2}\right)}\left(d y^{2}+d z^{2}\right)
$$

This demonstrates that in general, Eq. (22) corresponds to a conformally flat, homogeneous plane wave. It reduces to the Milne form of flat space only in the orthogonal model, where $\beta=0$, and only in this special case does the dimensionless shear parameter, $\Sigma_{+}$, vanish.

It is also important to consider the effects that spatial inhomogeneity may have on the possible set of initial conditions. The simplest class of inhomogeneous models are those that break homogeneity in only one direction $(x)$. In general, these models admit an abelian group of isometries, $G_{2}$, with orbits corresponding to space-like two-surfaces. (For a review, see, e.g., Ref. [23]). In the case where the $G_{2}$ admits two hypersurface-orthogonal Killing vector fields, the line-element may be written in the diagonal form [23]:

$$
d s^{2}=e^{f}\left(-d \xi^{2}+d x^{2}\right)+R\left(e^{p} d y^{2}+e^{-p} d z^{2}\right),
$$

where $f=f(\xi, x)$ and $p=p(\xi, x)$ represent the longitudinal and transverse parts of the gravitational field, respectively. The gradient of the function $R=R(\xi, x)$ determines the properties of the model and the Killing vectors are $\partial / \partial y$ and $\partial / \partial z$.

As an example, we consider the class of spatially compact cosmologies with a three-torus topology $S^{1} \times S^{1} \times S^{1}$. In this case, one may specify $R=\xi$ without loss of generality. The general solution to the Einstein-scalar field equations for these models has been found previously by Charach and Malin [24], who also discussed the asymptotic behaviour of the solution in the late time (high-frequency) limit, $\xi \rightarrow \infty$. In general, the scalar field, $\phi$, and transverse mode, $p$, decouple and both satisfy the one-dimensional cylindrical wave equation. In particular, the transverse part of the metric is given by 24]

$$
p=p_{0}+\alpha_{0} \ln \xi+\sum_{n=1}^{\infty} \cos \left[n\left(x-x_{n}\right)\right]\left[A_{n} J_{0}(n \xi)+B_{n} N_{0}(n \xi)\right],
$$

where $\left\{p_{0}, \alpha_{0}, A_{n}, B_{n}, x_{n}\right\}$ are arbitrary constants and $\left\{J_{0}, N_{0}\right\}$ are Bessel and Neumann functions of order zero. Formally, a similar expression may be written for the scalar field and the general form of the longitudinal mode, $f$, can then be determined.

It was found that the model asymptotically evolves into the Doroshkevich, Zeldovich and Novikov (DZN) universe [25]. This corresponds to an anisotropic, spatially homogeneous background with a null fluid composed of collisionless flows of massless scalar particles and gravitons. The late-time limit of the line-element is given in synchronous coordinates by

$$
d s^{2}=-d t^{2}+a_{1}^{2}(t) d x^{2}+a_{2}^{2}(t) d y^{2}+a_{3}^{2}(t) d z^{2}
$$


where

$$
a_{1} \propto t, \quad a_{2} \propto(\ln t)^{\left(\alpha_{0}+1\right) / 2}, \quad a_{3} \propto(\ln t)^{\left(1-\alpha_{0}\right) / 2}
$$

and Eq. (27) does not reduce to the Taub form of flat space, $d s^{2}=-d t^{2}+t^{2} d x^{2}+$ $d y^{2}+d z^{2}$.

In conclusion, we have argued that the recent conjecture that the Milne universe is a past asymptotic attractor for pre-big-bang cosmologies is a consequence of studying the dynamics in the synchronous frame. To substantiate this, we have provided a number of examples for which the past attractor is not the Milne form of flat space as given by Eq. (四). This included the class of orthogonal, anisotropic Bianchi B models. This class has a non-zero measure in the space of homogeneous initial data. A plane wave background is the attractor with a full measure of initial states for this class. Even in the Bianchi V case, where the orthogonal solution does tend towards Milne, the inclusion of tilt in the fluid flow removes this possibility. In addition, we have discussed the $G_{2}$ inhomogeneous generalization of the Bianchi I model for which the attractor is the homogeneous DZN cosmology.

These examples demonstrate that within the classes of models we have considered, the Milne universe is an unlikely past attractor for the pre-big-bang scenario. It should be emphasized that this does not indicate that such a state is not allowed and, indeed, it has a number of attractive features. However, we have provided quantitative arguments that suggest it is not generic. Moreover, our conclusions clearly do not constitute a general result within all possible models. In view of this, it would be interesting to consider other classes of inhomogeneous models to establish the extent of this likelihood in more general settings. Work in this direction is in progress.

Finally, in the above analysis of the orthogonal Bianchi models, we assumed that the axion field was homogeneous. Alternatively, one may consider the case where the two-form potential is homogeneous, which then implies an inhomogeneous axion field. Recently, Barrow and Kunze considered the most general form of the anti-symmetric field strength compatible with spatial homogeneity in this latter case [26]. They demonstrated that the Bianchi class B types III and $\mathrm{VI}_{h=0,-1 / 2,-2}$ are the most general. This is in marked contrast to the case where the axion field is homogeneous, where the types $\mathrm{VI}_{h}, \mathrm{VII}_{h}$, VIII and IX have the highest dimension. This would seem to indicate that assuming a time-dependent two-form potential could lead to different conclusions regarding the generality of the past attractor for spatially homogeneous pre-big-bang cosmologies. It would be interesting to investigate this possibility further.

\section{Acknowledgments}

D. C. and J. E. L. are supported by the Particle Physics and Astronomy Research Council (PPARC), U. K. and R. T. benefited from PPARC UK Grant No. L39094. We thank A. Feinstein, J. Maharana, G. Veneziano and J. Wainwright, for helpful communications. 


\section{References}

1. G. W. Gibbons, Quantum Gravity/String/M-theory as we approach the 3rd Millennium, gr-qc/9803065; M. J. Duff, "A Layman's Guide to M-theory", hep-th/9805177.

2. G. Veneziano, Phys. Lett. B 265, 287 (1991); M. Gasperini and G. Veneziano, Astropart. Phys. 1, 317 (1993); M. Gasperini and G. Veneziano, Mod. Phys. Lett. A8, 3701 (1993); M. Gasperini and G. Veneziano, Phys. Rev. D50, 2519 (1994).

3. R. Brustein and G. Veneziano, Phys. Lett. B 329, 429 (1994).

4. G. Veneziano, Phys. Lett. B 406, 297 (1997).

5. A. Buonanno, K. A. Meissner, C. Ungarelli, and G. Veneziano, Phys. Rev. D57, 2543 (1998).

6. M. S. Turner and E. J. Weinberg, Phys. Rev. D56, 4604 (1997); N. Kaloper, A. Linde, and R. Bousso, "Pre-big-bang requires the universe to be exponentially large from the very beginning", hep-th/9801073; J. Maharana, E. Onofri, and G. Veneziano, "A numerical simulation of pre-big-bang cosmology", hepth/9802001.

7. M. B. Green, J. H. Schwarz, and E. Witten, Superstring Theory: Vol. 2!x (Cambridge University Press, 1987).

8. L. D. Landau and E. M. Lifshitz, The Classical Theory of Fields (Pergamon Press, 1975).

9. M. P. Ryan and L. C. Shepley, Homogeneous Relativistic Cosmologies (Princeton University Press, Princeton, 1975).

10. G. F. R. Ellis and M. A. H. MacCallum, Commun. Math. Phys. 12, 108 (1969).

11. C. B. Collins and S. W. Hawking, Astrophys. J. 180, 317 (1973).

12. C. G. Hewitt and J. Wainwright, Class. Quantum Grav. 10, 99 (1993).

13. K. C. Jacobs, Astrophys. J. 153, 661 (1968).

14. E. Kasner, Trans. Am. Math. Soc. 27, 155 (1925).

15. D. Clancy, J. E. Lidsey, and R. Tavakol, "Effects of anisotropy and spatial curvature on the pre-big-bang scenario", gr-qc/9802052, Phys. Rev. D to be published. 
16. S. T. C. Siklos, Class. Quantum Grav. 8, 1587 (1991).

17. J. D. Barrow and D. Sonada, Phys. Rep. 139, 1 (1986).

18. A. G. Doroshkevich, V. N. Lukash, and I. D. Novikov, Sov. Phys. JETP 37, 739 (1973).

19. V. N. Lukash, Sov. Phys. JETP 40, 792 (1975).

20. J. D. Barrow, Nat. 272, 211 (1977).

21. C. G. Hewitt and J. Wainwright, Phys. Rev. D46, 4242 (1992).

22. R. Maartens and S. D. Nel, "Spatially homogeneous locally rotationally symmetric cosmological models", (B.Sc. (Hons) thesis, University of Cape Town, 1976); J. Wainwright, W. C. W. Ince, and B. J. Marshman, Gen. Rel. Grav. 10, 259 (1979).

23. M. Carmeli, Ch. Charach, and S. Malin, Phys. Rep. 76, 79 (1981).

24. Ch. Charach and S. Malin, Phys. Rev. D19, 1058 (1979).

25. A. G. Doroshkevich, Y. B. Zeldovich, and I. D. Novikov, Sov. Phys. JETP 26, 408 (1968).

26. J. D. Barrow and K. E. Kunze, Phys. Rev. D55, 623 (1997). 